\title{
Bifurcation Behavior Analysis in a Predator-Prey Model
}

\author{
Nan Wang, ${ }^{1,2}$ Min Zhao, ${ }^{2,3}$ Hengguo Yu, ${ }^{1,2}$ Chuanjun Dai,, \\ Beibei Wang, ${ }^{1,2}$ and Pengfei Wang ${ }^{1,2}$ \\ ${ }^{1}$ School of Mathematics and Information Science, Wenzhou University, Wenzhou, Zhejiang 325035, China \\ ${ }^{2}$ Zhejiang Provincial Key Laboratory for Water Environment and Marine Biological Resources Protection, \\ Wenzhou University, Wenzhou, Zhejiang 325035, China \\ ${ }^{3}$ School of Life and Environmental Science, Wenzhou University, Wenzhou, Zhejiang 325035, China
}

Correspondence should be addressed to Min Zhao; zmcn@tom.com

Received 24 October 2015; Accepted 4 January 2016

Academic Editor: Taher S. Hassan

Copyright (C) 2016 Nan Wang et al. This is an open access article distributed under the Creative Commons Attribution License, which permits unrestricted use, distribution, and reproduction in any medium, provided the original work is properly cited.

A predator-prey model is studied mathematically and numerically. The aim is to explore how some key factors influence dynamic evolutionary mechanism of steady conversion and bifurcation behavior in predator-prey model. The theoretical works have been pursuing the investigation of the existence and stability of the equilibria, as well as the occurrence of bifurcation behaviors (transcritical bifurcation, saddle-node bifurcation, and Hopf bifurcation), which can deduce a standard parameter controlled relationship and in turn provide a theoretical basis for the numerical simulation. Numerical analysis ensures reliability of the theoretical results and illustrates that three stable equilibria will arise simultaneously in the model. It testifies the existence of Bogdanov-Takens bifurcation, too. It should also be stressed that the dynamic evolutionary mechanism of steady conversion and bifurcation behavior mainly depend on a specific key parameter. In a word, all these results are expected to be of use in the study of the dynamic complexity of ecosystems.

\section{Introduction}

The dynamical behaviors between different populations and their complex properties have been given close attention by biologists and ecologists. Since the pioneering work of Lotka and Volterra, the research interest in predator-prey dynamics has achieved constant attention. It is well known that these models can directly reflect changes in the size of populations. Considerable improvements are that the relevant theories become more and more complete in this category in recent years [1-5].

The predator-prey models have extensive applicability in the field of biological problems. The biologist can use them to study the relationship between species in different domains [6-10]. Yang and Zhao [11] have established a fish-algae consumption model to explore how to apply the complex dynamics between fish-algae populations to expound the mechanism of algae blooms; these results will be helpful in controlling algae bloom. González-Olivares and Rojas-Palma [12] have established a Gause type predator-prey model with Allee effects and considered three standard functional responses, respectively. They found that different types of functional responses will lead to a model's dynamic behavior change. Their results perfectly explore that the expression of one interaction term has a significant effect on the stability and persistence of the population model, which is meaningful in establishing biological mathematical model. Dhar et al. [13] have proposed a mathematical model to study how instantaneous nutrient recycling affects the dynamic characteristic of aquatic ecosystem. The nutrient supply rate was found to affect the local stability of equilibrium; this work was significant for models involving flowing waters. Luo [14] has considered a mathematical model to study how the periodic environment influences the internal operating characteristics of the aquatic ecosystem. He pointed out that the temperature has a certain time periodicity, the fluctuation of temperature can lead to changes in intrinsic carrying capacity, and the growth rate of prey populations can also be influenced by time periodicity; these results in [14] are more accordant with the actual situation. 
It is a common phenomenon in nature that one predator lives on multiple prey species. To address this issue, some researchers have begun to consider alternative prey to depict the dynamic predator mechanism [15-18]. It is easy to find out that alternative prey can variously affect the dynamic capture feature. On one hand, alternative prey can increase the predation quantity for the focal prey, because more prey biomass may result in higher predation rates for both prey items. On the other hand, the alternative prey population can also lower predation on the focal prey because of predator preference for the alternative prey resources [19]. At the same time, many studies show that the alternative population can intensively influence the dynamic behavior of the aquatic ecosystem $[20,21]$. Based on this mechanism, Kar and Chattopadhyay [22] have developed a two-species predator-prey model which includes the effect of alternative prey. The model can be depicted as

$$
\begin{aligned}
& \frac{d N}{d T}=r_{1} N\left(1-\frac{N}{k_{1}}\right)-\frac{a_{1} N}{b_{1}+N} P, \\
& \frac{d P}{d T}=\frac{a_{1} e_{1} N}{b_{1}+N} P+s_{1} P\left(1-\frac{N}{k_{1}}\right)-d_{1} P,
\end{aligned}
$$

where $N$ and $P$ represent prey and predator population densities or biomass at time $T$, respectively. Here, $r_{1}$ stands for the intrinsic growth rate of the prey without any environment limitations, $k_{1}$ is the environmental carrying capacity of the prey in the absence of the predator, $a_{1} N /\left(b_{1}+N\right)$ is the Holling type-II functional response [23], which is used to depict the average feeding rate of the predator when the predator spends time seeking prey, where $b_{1}$ is the half saturation constant for the Holling type-II, $a_{1}$ is the grazing rate of the predator population, $e_{1}$ and $d_{1}$ are the conversional rate and mortality rate of predator, respectively, and $s_{1} P\left(1-N / k_{1}\right)$ indicates the portion of biomass of predator increments from the alternative prey, where $s_{1}$ represents the growth rate of the predator on account of the alternative prey. From the formula, we can see that when the quantity of focal prey $N$ approaches the environmental carrying capacity $k_{1}$, the amount of alternative prey consumed by the predator will tend to be zero [24].

The concept of Allee effect was firstly derived from the research of Allee and Bowen [25]. Since then, the Allee effect received the attention of many researchers [26-29]. Allee effects can be roughly classified into two types: strong and weak [30]. For these two forms, there is a critical value that is referred to as the Allee threshold, respectively. The fist form means that if the population size is below the threshold, the species will become extinct. When the growth rate gradually decreases but remains positive with a low population size, the Allee effect is described as weak. Aulisa and Jang [31] have established a continuous-time predator-prey model to study influences in dynamical behaviors when the prey population possesses Allee effect. They pointed out that both species will become extinct if the prey population size falls below a certain threshold. Pan et al. [32] have considered a reaction-diffusion phytoplankton-zooplankton model with double Allee effects on prey population. They pointed out that the Allee effects can make the dynamical behaviors of a system increasingly complex. form:

The strong Allee effect can be depicted by the following

$$
\frac{d N}{d T}=r_{1} N\left(1-\frac{N}{k_{1}}\right)\left(\frac{N}{m_{1}}-1\right),
$$

where $m_{1}$ is Allee effect threshold. If population density or size is below the threshold, this population is doomed to extinction. Here $0<m_{1}<k_{1}$, the other parameters' significance is the same as model (1).

Now we will establish a predator-prey model with strong Allee effects:

$$
\begin{aligned}
& \frac{d N}{d T}=r_{1} N\left(1-\frac{N}{k_{1}}\right)\left(\frac{N}{m_{1}}-1\right)-\frac{a_{1} N}{b_{1}+N} P, \\
& \frac{d P}{d T}=\frac{a_{1} e_{1} N}{b_{1}+N} P+s_{1} P\left(1-\frac{N}{k_{1}}\right)-d_{1} P .
\end{aligned}
$$

The parameters are all greater than zero and have the same significance as above. For simplicity, we write the above model in dimensionless form as follows: we take the scaling $N=k_{1} n, P=r_{1} p / a_{1}$, and $T=t / r_{1}$; then, model (3) can be simplified as

$$
\begin{aligned}
& \frac{d n}{d t}=n(1-n)(m n-1)-\frac{n}{b+n} p=F_{1}, \\
& \frac{d p}{d t}=\frac{e n}{b+n} p+s p(1-n)-d p=F_{2},
\end{aligned}
$$

where $e=a_{1} e_{1} / r_{1}, b=b_{1} / k_{1}, s=s_{1} / r_{1}, d=d_{1} / r_{1}, m=$ $k_{1} / m_{1}$, and apparently $m>1$.

In the succedent subsections of this paper, we introduce the problem of determining the number of equilibria. Stability analysis of equilibrium point is also presented. Then, we provide a demonstration of several types of bifurcation and give a set of parameter values to prove the existence of Bogdanov-Takens (BT) bifurcation. In Section 3, numerical simulations are given to illustrate the theoretical analysis results, followed by conclusions in Section 4.

\section{Qualitative Analysis}

2.1. Equilibria. In this subsection, we mainly concentrate on the existence of positive equilibrium of model (4a) and (4b). Model (4a) and (4b) has three boundary equilibria: $E_{0}(0,0)$, $E_{1}(1,0)$, and $E_{2}(1 / m, 0)$ and they are always existent. The interior equilibria are the intersection points of the vertical isocline and horizontal isocline in the interior of the first quadrant. The expressions of vertical and horizontal isoclines are as follows:

$$
\begin{aligned}
(1-n)(m n-1)-\frac{p}{b+n} & =0, \\
\frac{e n}{b+n}+s(1-n)-d & =0 .
\end{aligned}
$$

The horizontal isocline is vertical line and the number of perpendiculars is decided by (5b). We denote (5b) by $H(n)=$ $G(n) /(b+n)=0$, where $G(n)=-s n^{2}+(e+s-s b-d) n+b(s-d)$. 
$G(n)=0$ have the same solutions as (5b), and two solutions exist in the equation at most. We denoted them by $n_{3}$ and $n_{4}$, where $n_{4}<n_{3}$. From the geometric properties of isoclines we can know that model (4a) and (4b) has interior equilibrium points if and only if $1 / m<n_{3}<1$ or $1 / m<n_{4}<1$. Then, the maximum number of equilibria for model (4a) and (4b) is five. We use $E_{3}\left(n_{3}, p_{3}\right)$ and $E_{4}\left(n_{4}, p_{4}\right)$ to signify the interior equilibria, the existence and stability of which are shown as follows.

The Jacobian matrix of model (4a) and (4b) at the equilibrium point $E_{i}$ is

$$
\begin{aligned}
J_{\left(n_{i}, p_{i}\right)} & =\left(\begin{array}{ll}
A\left(n_{i}, p_{i}\right) & B\left(n_{i}, p_{i}\right) \\
C\left(n_{i}, p_{i}\right) & D\left(n_{i}, p_{i}\right)
\end{array}\right), \\
A\left(n_{i}, p_{i}\right) & =-3 m n_{i}^{2}+(2 m+2) n_{i}-1-\frac{b p_{i}}{\left(b+n_{i}\right)^{2}}, \\
B\left(n_{i}, p_{i}\right) & =-\frac{n_{i}}{b+n_{i}}, \\
C\left(n_{i}, p_{i}\right) & =\frac{b e p_{i}}{\left(b+n_{i}\right)^{2}}-s p_{i}, \\
D\left(n_{i}, p_{i}\right) & =\frac{e n_{i}}{b+n_{i}}+s-d-s n_{i} .
\end{aligned}
$$

Theorem 1. (1) $E_{0}(0,0)$ is an asymptotically stable node point if $s<d$ and a saddle point for $s>d$; if $s=d$, it is a high order singularity. The Jacobian matrix around $E_{0}$ shows that

$$
J_{(0,0)}=\left(\begin{array}{cc}
-1 & 0 \\
0 & s-d
\end{array}\right) \text {. }
$$

(2) $E_{1}(1,0)$ is a asymptotically stable node point when e/(b+ $1)<d$ while it is a saddle point when $e /(b+1)>d$. The Jacobian matrix around $E_{1}$ is

$$
J_{(1,0)}=\left(\begin{array}{cc}
1-m & -\frac{1}{b+1} \\
0 & \frac{e^{b+1}-d}{b+1}
\end{array}\right) \text {. }
$$

(3) $E_{2}(1 / m, 0)$ is always unstable. The Jacobian matrix around $E_{2}$ is

$$
J_{(1 / m, 0)}=\left(\begin{array}{cc}
1-\frac{1}{m} & e^{-\frac{1}{b m+1}} \\
0 & \frac{e^{b m+1}+s-d-\frac{s}{m}}{b m}
\end{array}\right)
$$

One of the eigenvalues is $1-1 / m>0$, so $E_{2}$ is unstable.

Theorem 2. If $e-d-b d>0$ and $-s / m^{2}+(e+s-s b-$ $d) / m+b(s-d)<0$ hold, model (4a) and (4b) has only one interior equilibrium $E_{3}\left(n_{3}, p_{3}\right)$. Furthermore, $E_{3}\left(n_{3}, p_{3}\right)$ is always unstable, especially when $d=s+e+s b-2 \sqrt{b s e}$; it is a high-order singularity, where

$$
\begin{aligned}
& n_{3} \\
& =\frac{s+e-s b-d+\sqrt{(s b-s+d-e)^{2}+4 b s(s-d)}}{2 s}, \\
& p_{3}=\left(1-n_{3}\right)\left(m n_{3}-1\right)\left(b+n_{3}\right) .
\end{aligned}
$$

Proof. According to (6),

$$
\begin{aligned}
\operatorname{det}\left(J_{\left(n_{3}, p_{3}\right)}\right) & =-C\left(n_{3}, p_{3}\right) B\left(n_{3}, p_{3}\right) \\
& =\frac{n_{3} p_{3}}{b+n_{3}}\left(\frac{b e}{\left(b+n_{3}\right)^{2}}-s\right) .
\end{aligned}
$$

Let $\phi=n_{3}-\sqrt{b e / s}+b$; substituting (11a) into $\phi$, we get

$\phi$

$$
\begin{aligned}
& =\frac{s+e-d+s b-2 \sqrt{b e s}+\sqrt{(s+e-d-s b)^{2}+4 b s(s-d)}}{2 s} \\
& =\frac{s+e-d+s b-2 \sqrt{b e s}+\sqrt{(s+e-d+s b)^{2}-4 b s e}}{2 s} \geq 0 .
\end{aligned}
$$

Then, we know when $n_{3} \geq \sqrt{b e / s}-b$ and $\operatorname{det} J_{\left(n_{3}, p_{3}\right)} \leq 0$, $E_{3}$ is a saddle point or a high-order singularity.

Theorem 3. If $e-d-b d<0$ and $-s / m^{2}+(e+s-s b-d) / m+b(s-$ d) $>0$, model (4a) and (4b) has only one interior equilibrium $E_{4}\left(n_{4}, p_{4}\right)$. Furthermore, $E_{4}\left(n_{4}, p_{4}\right)$ is locally asymptotically stable when $n_{4} \in L$, where $L=\{n \mid A(n)<0, n \in(1 / m, 1)\}$ and

$n_{4}$

$$
\begin{aligned}
& \quad=\frac{s+e-s b-d-\sqrt{(s b-s+d-e)^{2}+4 b s(s-d)}}{2 s}, \\
& p_{4}=\left(1-n_{4}\right)\left(m n_{4}-1\right)\left(b+n_{4}\right) .
\end{aligned}
$$

Proof. According to (6),

$$
\begin{aligned}
\operatorname{trace}\left(J_{\left(n_{4}, p_{4}\right)}\right)= & A\left(n_{4}\right) \\
= & -3 m n_{4}^{2}+(2 m+2) n_{4}-1 \\
& -\frac{b\left(1-n_{4}\right)\left(m n_{4}-1\right)}{b+n_{4}}, \\
\operatorname{det}\left(J_{\left(n_{4}, p_{4}\right)}\right)= & -C\left(n_{4}\right) B\left(n_{4}\right) \\
= & \frac{n_{4}}{b+n_{4}}\left(\frac{b e p_{4}}{\left(b+n_{4}\right)^{2}}-s p_{4}\right) .
\end{aligned}
$$

Imitating the proof of Theorem 2, we can know

$$
n_{4}<\sqrt{\frac{b e}{s}}-b ;
$$


then, $\operatorname{det}\left(J_{\left(n_{4}, p_{4}\right)}\right)=-B\left(n_{4}\right) C\left(n_{4}\right)>0$, which means two eigenvalues have the same sign.

Since

$$
\begin{aligned}
A\left(\frac{1}{m}\right) & =1-\frac{1}{m}>0, \\
A(1) & =1-m<0 .
\end{aligned}
$$

Solving $A(n)=0$, we can get that there exists a set $L \subset$ $(1 / m, 1)$, such that $A(n)<0$ for all $n \in L$, where $L=$ $\left(\left(1+m-m b+\sqrt{1-m+m b+m^{2}\left(1+b+b^{2}\right)}\right) / 3 m, 1\right)$. Then, we know that if $n_{4} \in L$, trace $\left(J_{\left(n_{4}, p_{4}\right)}\right)=A\left(n_{4}\right)<0$ and both eigenvalues of $J_{\left(n_{4}, p_{4}\right)}$ have negative real parts, and $E_{4}\left(n_{4}, p_{4}\right)$ is locally asymptotically stable.

From the above discussion, we find that model (4a) and (4b) may possess two interior equilibria $E_{3}$ and $E_{4}$ simultaneously if $e-d-b d<0,-s / m^{2}+(e+s-s b-$ $s) / m+b(s-d)<0,(s b-s+d-e)^{2}+4 b s(s-d)>0$, and $2 s / m<e+s-s b-d<2 s$. The stability condition of $E_{4}$ is still $n_{4} \in L$.

Considering the existence and stability conditions integrated, we know that there will appear three stable equilibria synchronously if the scope of those parameters satisfies the above conditions. Based on the above demonstration, some cases where the stability of equilibria may occur are cited in Table 1, where the existence of equilibria is classified according to the magnitude of $s$ and $d$.

2.2. Local Bifurcation. From Table 1 we know that the variation of parameter value will lead to the number change of interior equilibria. We take $d$ as variable parameter and find that changing the value of $d$ will vary equilibrium's number; when the value of $d$ increases across the threshold $d_{\mathrm{TC} 1}=$ $e /(b+1)$, the interior equilibrium $E_{4}$ bifurcates from $E_{1}$, and when the value of $d$ is across $d_{\mathrm{TC} 2}=e /(b m+1)+s(1-1 / m)$, another interior equilibrium $E_{3}$ can bifurcate from $E_{2}$. Then, there exist two transcritical bifurcations, which are denoted by $\mathrm{TC} 1$ and TC2, respectively.

Theorem 4. (1) Model (4a) and (4b) undergoes transcritical bifurcation at $E_{1}(1,0)$ when the value of parameter $d$ equals the transcritical bifurcation threshold $d_{T C 1}=e /(b+1)$.

(2) Model (4a) and (4b) undergoes another transcritical bifurcation at $E_{2}(1 / m, 0)$ when the value of parameterd equals the transcritical bifurcation threshold $d_{T C 2}=e /(b m+1)+s(1-$ $1 / m)$.

Proof. It can be easily seen that $E_{1}(1,0)$ coincides with $E_{4}\left(n_{4}, p_{4}\right)$ when $d=e /(b+1)$. According to the theorems in $[33,34]$, it can be found that one interior equilibrium point branches off from $E_{1}$ when $d$ passes the threshold $d_{\mathrm{TC} 1}=$ $e /(b+1)$ and also conforms with the transversality condition for transcritical bifurcation. The same notation we followed in this paper is mentioned in [33].

We can calculate the value of Jacobian matrix of model (4a) and $(4 \mathrm{~b})$ as $\left.\operatorname{Det}\left(J_{(1,0)}\right)\right|_{d_{\mathrm{TCl}}}$, and then $(1,0)$ is a nonhyperbolic equilibrium point when $d=d_{\mathrm{TC} 1}$. Let the eigenvectors $v=[1,(b+1)(1-m)]^{T}$ and $w=[0,1]^{T}$ indicate the eigenvectors corresponding to zero eigenvalues of $J_{\left((1,0), d_{\mathrm{TC} 1}\right)}$ and
$\left[J_{\left((1,0), d_{\mathrm{TC1}}\right)}\right]^{T}$, respectively. Next the transversality conditions for the transcritical bifurcation are satisfied be verified, where $F=\left(F_{1}, F_{2}\right)^{T}$ :

$$
\begin{aligned}
& w^{T} F_{d}\left((1,0) ; d_{\mathrm{TC} 1}\right)=(0,1)(0,0)^{T}=0, \\
& w^{T} D F_{d}\left((1,0) ; d_{\mathrm{TC} 1}\right) v=(b+1)(m-1) \neq 0, \\
& w^{T} D^{2} F\left((1,0) ; d_{\mathrm{TC} 1}\right)(v, v) \\
& =\left(\frac{e b}{(1+b)^{2}}-s\right)(b+1)(1-m) .
\end{aligned}
$$

We notice that $m>1$ and $w^{T} D^{2} F\left((1,0) ; d_{\mathrm{TC} 1}\right)(v, v)<$ 0 if $e>s(b+1)^{2} / b$ and then the transcritical bifurcation is supercritical, which means that an interior equilibrium point arises through $E_{1}$ under this condition. Another aspect is that if $e<s(b+1)^{2} / b$, the transcritical bifurcation is subcritical and an interior equilibrium vanishes across $E_{1}$.

In the following, we demonstrate that another interior equilibrium point bifurcates from $E_{2}(1 / m, 0)$ through transcritical bifurcation at the threshold $d_{\mathrm{TC} 2}=e /(b m+1)+$ $s(1-1 / m)$. Similarly, we can get $\left.\operatorname{Det}\left(J_{(1 / m, 0)}\right)\right|_{d_{\mathrm{TC} 2}}=0, v=$ $[1,(b+1 / m)(m-1)]^{T}$, and $w=[0,1]^{T}$ are the eigenvectors corresponding to $J_{\left((1 / m, 0), d_{\mathrm{TC} 2}\right)}$ and $\left[J_{\left((1 / m, 0), d_{\mathrm{TC} 2}\right)}\right]^{T}$, respectively. We have

$$
\begin{aligned}
& w^{T} F_{d}\left(\left(\frac{1}{m}, 0\right) ; d_{\mathrm{TC} 2}\right)=(0,1)(0,0)^{T}=0, \\
& w^{T} D F_{d}\left(\left(\frac{1}{m}, 0\right) ; d_{\mathrm{TC} 2}\right) v=(1-m)\left(b+\frac{1}{m}\right) \neq 0, \\
& w^{T} D^{2} F\left(\left(\frac{1}{m}, 0\right) ; d_{\mathrm{TC} 2}\right)(v, v) \\
& =\left(\frac{b e}{(b+1 / m)^{2}}-s\right)\left(b+\frac{1}{m}\right)(m-1) .
\end{aligned}
$$

If $e<s(b+1 / m)^{2} / b$ and $w^{T} D^{2} F\left((1 / m, 0) ; d_{\mathrm{TC} 2}\right)(v, v)<0$, the transcritical bifurcation is supercritical and an interior equilibrium point appears across $E_{2}$ under this condition. Another aspect is that if $e>s(b+1 / m)^{2} / b$, the transcritical bifurcation is subcritical and an interior equilibrium vanishes across $E_{2}$.

Theorem 5. Model (4a) and (4b) undergoes saddle-node bifurcation when $d=d_{S N}$, where $d_{S N}=s+e+b s-2 \sqrt{b s e}$.

Proof. It is easy to calculate that the discriminant of $G(n)=0$ is equal to zero when $d=s+e+b s-2 \sqrt{b s e}$, which means $G(n)=0$ has a twofold root. We denote this root by $n^{*}$. Model (4a) and (4b) has only one interior equilibrium point $E^{*}\left(n^{*}, p^{*}\right)$ correspondingly and the constituents are given by

$$
\begin{aligned}
& n^{*}=\sqrt{\frac{e b}{s}}-b, \\
& p^{*}=\left(1-n^{*}\right)\left(m n^{*}-1\right)\left(b+n^{*}\right) .
\end{aligned}
$$


TABLE 1

\begin{tabular}{|c|c|c|c|c|c|c|}
\hline & & $E_{0}(0,0)$ & $E_{1}(1,0)$ & $E_{2}(1 / m, 0)$ & $E_{3}\left(n_{3}, p_{3}\right)$ & $E_{4}\left(n_{4}, p_{4}\right)$ \\
\hline & $s>d$ & Saddle point & Stable node point & Unstable node point & Saddle point & Nonexistent \\
\hline & $s=d$ & $\begin{array}{c}\text { Unstable } \\
\text { (high-order } \\
\text { singularity) }\end{array}$ & Stable node point & Unstable node point & Saddle point & Nonexistent \\
\hline \multirow{4}{*}{$s<d$} & $\Delta=0$ & Stable node point & Stable node point & Saddle point & \multicolumn{2}{|c|}{$E_{3}$ and $E_{4}$ coincide and are a saddle-node point } \\
\hline & \multirow{3}{*}{$\Delta>0$} & Stable node point & Stable node point & Unstable node point & Saddle point & Nonexistent \\
\hline & & Stable node point & Saddle point & Saddle point & Nonexistent & Stable node point \\
\hline & & Stable node point & Stable node point & Saddle point & Saddle point & Stable node point \\
\hline
\end{tabular}

The Jacobian matrix evaluate at $E^{*}$ is given by

$$
\begin{aligned}
J_{\left(\left(n^{*}, p^{*}\right), d_{\mathrm{SN}}\right)} & =\left(\begin{array}{cc}
A\left(n^{*}, p^{*}, d_{\mathrm{SN}}\right) & B\left(n^{*}, p^{*}, d_{\mathrm{SN}}\right) \\
C\left(n^{*}, p^{*}, d_{\mathrm{SN}}\right) & D\left(n^{*}, p^{*}, d_{\mathrm{SN}}\right)
\end{array}\right) \\
& =\left(\begin{array}{cc}
M & \sqrt{\frac{b s}{e}}-1 \\
0 & 0
\end{array}\right),
\end{aligned}
$$

where $M=A\left(n^{*}, p^{*}, d_{\mathrm{SN}}\right)$. Obviously, matrix $J_{\left(\left(n^{*}, p^{*}\right), d_{\mathrm{SN}}\right)}$ has a double zero eigenvalues when $\operatorname{Det}\left(J_{\left(\left(n^{*}, p^{*}\right), d_{\mathrm{SN}}\right)}\right)=0$. We can count out the eigenvectors corresponding to the zero eigenvalue, which are $v=[1, M /(1-\sqrt{b s / e})]^{T}$ and $w=$ $[0,1]^{T}$. Utilizing the expressions of vectors $v$ and $w$, as well as $n^{*} \in(1 / m, 1)$, we can get

$$
\begin{aligned}
& w^{T} F_{d}\left(\left(n^{*}, p^{*}\right) ; d_{\mathrm{SN}}\right) \\
& =-\left(1-n^{*}\right)\left(m n^{*}-1\right)\left(b+n^{*}\right)<0, \\
& w^{T}\left[D^{2} F\left(\left(n^{*}, p^{*}\right) ; d_{\mathrm{SN}}\right)\right](v, v) \\
& =\frac{-e b\left(1-n^{*}\right)\left(m n^{*}-1\right)}{\left(b+n^{*}\right)^{2}}
\end{aligned}
$$

Given the value range of $n^{*}$, we know that $w^{T}\left[D^{2} F\left(\left(n^{*}, p^{*}\right) ; d_{\mathrm{SN}}\right)\right](v, v)$ cannot be equal to zero. Then, by Sotomayor's theorem we can prove that the model undergoes a saddle-node bifurcation when the parameter $d$ goes via the critical threshold $d=d_{\mathrm{SN}}$.

Theorem 6. In the case of $s<d$, the interior equilibrium point $E_{4}$ changes its stability through the Hopf-bifurcation at the threshold $b=b_{H}$, where $b_{H}=-\left(3 m n_{4}{ }^{2}-2(m+1) n_{4}+\right.$ 1)/ $\left(2 m n_{4}-m-1\right)$.

Proof. Because the interior equilibrium point $E_{3}$ is always a saddle, then Hopf bifurcation can only take place at $E_{4}$. Parameter $b$ can drive equilibrium $E_{4}$ into an unstable state when $b>b_{H}$, so $b=b_{H}$ is the critical value where the stability of $E_{4}$ changes. Next we will prove the necessary condition for Hopf bifurcation to occur. To testify the transversality condition of the Hopf bifurcation of the model's solution, we take $\lambda=\alpha(b)+\beta(b) i$, where $\lambda$ is an eigenvalue of the Jacobian matrix $J_{\left(\left(n_{4}, p_{4}\right), b_{H}\right)}$. If the Hopf bifurcation occurs at $b=b_{H}, \lambda$ is a purely imaginary number, such that $\operatorname{Tr}\left(J_{\left(\left(n_{4}, p_{4}\right), b_{H}\right)}\right)=0$, $\operatorname{Det}\left(J_{\left(\left(n_{4}, p_{4}\right), b_{H}\right)}\right) \neq 0$, and $d \operatorname{Tr}\left(J_{\left(\left(n_{4}, p_{4}\right), b_{H}\right)}\right) / d b \neq 0$. Substituting $b$ by $b_{H}=-\left(3 m n_{4}{ }^{2}-2(m+1) n_{4}+1\right) /\left(2 m n_{4}-m-1\right)$, we can get

$$
\begin{aligned}
& \operatorname{Tr}\left(J_{\left(n_{4}, p_{4}, b_{H}\right)}\right)=0, \\
& \operatorname{Det}\left(J_{\left(n_{4}, p_{4}, b_{H}\right)}\right) \\
& \quad=n_{4}\left(1-n_{4}\right)\left(m n_{4}-1\right)\left(\frac{b_{H} e}{\left(b_{H}+n_{4}\right)^{2}}-s\right), \\
& \frac{d}{d b} \operatorname{Tr}\left(J_{\left(n_{4}, p_{4}, b_{H}\right)}\right)=-\frac{n_{4}\left(1-n_{4}\right)\left(m n_{4}-1\right)}{\left(b+n_{4}\right)^{2}} .
\end{aligned}
$$

Using (16), we know $\operatorname{Det}\left(J_{\left(n_{4}, p_{4}, b_{H}\right)}\right)>0$ and $d \operatorname{Tr}\left(J_{\left(n_{4}, p_{4}, b_{H}\right)}\right) / d b \neq 0$. Then, the transversality condition of a Hopf bifurcation is satisfied [35].

Theorem 7. Model (4a) and (4b) undergoes a BogdanovTakens (BT) bifurcation of codimension two.

As we know, $\operatorname{Tr}(J)=0$ and $\operatorname{Det}(J)=0$ are the necessary conditions of the occurrence of Hopf and saddle-node, respectively. If both Hopf and saddle-node conditions hold, there will be a new bifurcation called Bogdanov-Takens bifurcation. In this situation, the Jacobian matrix has a double zero eigenvalue [36]. Since the explicitly analytical expressions for thresholds of BT bifurcation are quite difficult to determine, we give a numerical example to confirm the system exhibit BTs bifurcation. We fix $m=1.5, s=0.1$, and $d=0.4$. We find that, at $\left(e_{B T}, b_{B T}\right)=(0.4930734388,0.2386077213)$, $\left.\operatorname{Tr}\left(J_{E^{*}}\right)\right|_{\left(e_{B T}, b_{B T}\right)}=0$ and $\left.\operatorname{Det}\left(J_{E^{*}}\right)\right|_{\left(e_{B T}, b_{B T}\right)}=0$. Moreover, $\left(\partial^{2} F / \partial n^{2}-(\partial F / \partial n)\left(\partial^{2} F / \partial n \partial p\right)\right) /\left(\partial^{2} F / \partial n \partial p+\right.$ $\left.\partial^{2} F / \partial n \partial p\right)=-2.800482957$ and $\left((1 / 2)(\partial F / \partial n)\left(\partial^{2} F / \partial n^{2}\right)-\right.$ $\left.(\partial F / \partial n)^{2}\left(\partial^{2} F / \partial n \partial p\right)\right) /\left(\partial F / \partial p+(1 / 2)(\partial F / \partial p)\left(\partial^{2} F / \partial n^{2}\right)-\right.$ $\left.(\partial F / \partial n)\left(\partial^{2} F / \partial n \partial p\right)\right)=-0.000073428878$; these expressions prove the transversality conditions for a BT bifurcation [37].

\section{Numerical Results}

In order to verify the correctness and feasibility of the theoretical results, a series of numerical simulations will be depicted in detail. Many phase diagrams are given to display the dynamics properties of model (4a) and (4b), 


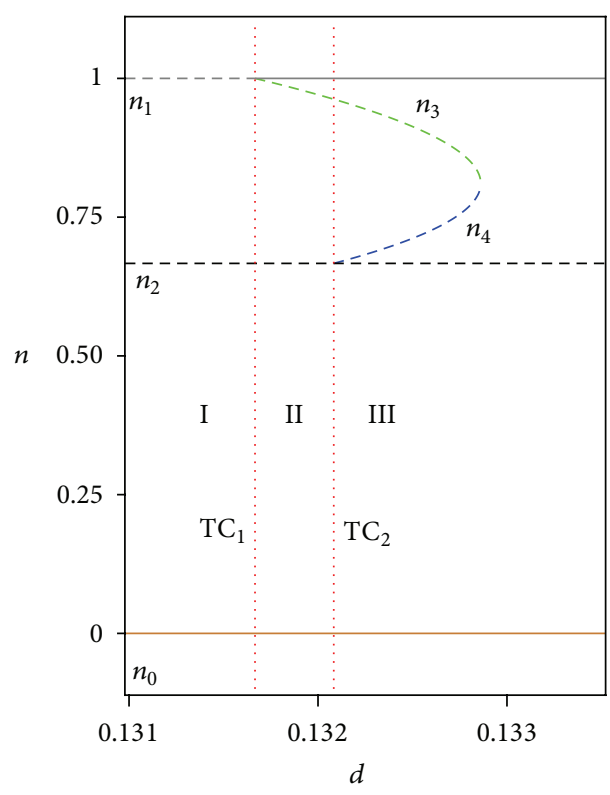

(a)

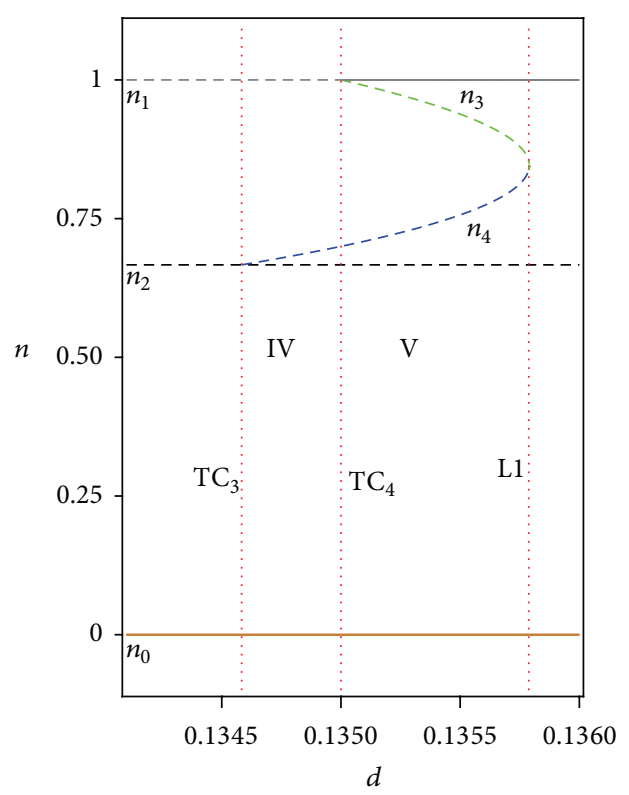

(b)

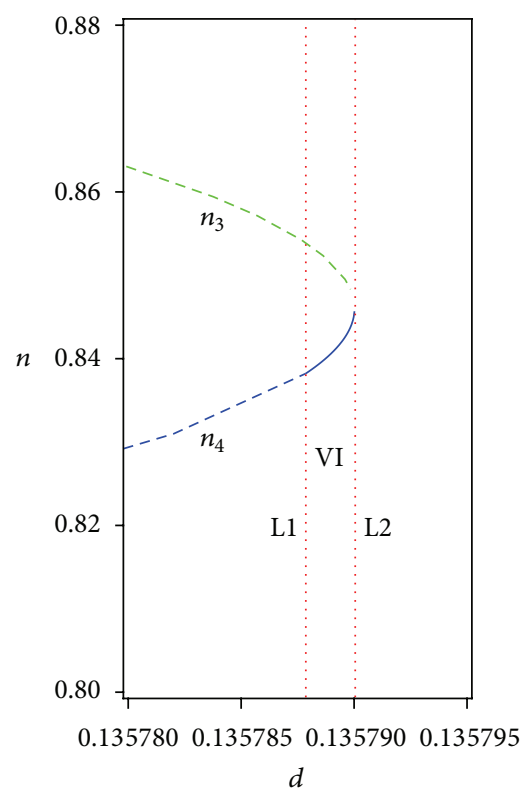

(c)

Figure 1: Bifurcation diagram of model (4a) and (4b) in $n-d$ plane constructed for $m=1.5, b=2.0, s=0.1, e=0.395$ (a), and $e=$ 0.405 (b, c). Red perpendicular dashed lines represent the critical value for different bifurcation occurrence. Three horizontal lines stand for three boundary equilibria: $E_{0}$ (golden), $E_{1}$ (gray), and $E_{2}$ (black). Two interior equilibria $E_{3}$ and $E_{4}$ are presented by blue and green curves, respectively. The solid lines indicate equilibrium in stable state and dotted line indicates unstable state, where, according to the stability theorem, these results are calculated and drawn based on Maple 14 platform.

which are based on pplane8 routines [38] in Matlab 7.1. In fact, according to [39], we can know pplane8 is a powerful tool for studying planar autonomous systems of differential equations, which can rapidly and accurately draw trajectories of each phase plane, count each critical point, and correctly characterize each equilibrium point of the studied systems. It is easy to find from Table 1 that model (4a) and (4b) only has an interior equilibrium point $E_{3}$ if $s \geq d$; then, the premise of parametric ranges in Figure 1 is $s>d$. It should be pointed out from Figure 1(a) that two vertical lines passing through points $(e /(b+1), 0)$ and $(e /(b m+1)+s(1-s / m), 0)$ are the transcritical bifurcation curves, which have been named TC1 and TC2, respectively. Furthermore, if $d<e /(b+1)$ is feasible, that is to say, model (4a) and (4b) does not have any interior equilibrium point when the value of $d$ is positioned within region I of Figure 1(a), three boundary equilibria $E_{0}(0,0)$, 
$E_{1}(1,0)$, and $E_{2}(2 / 3,0)$ exist. $E_{0}(0,0)$ is asymptotically stable, while $E_{1}(1,0)$ and $E_{2}(2 / 3,0)$ are unstable, the results of which have been shown in Figure 2(a) $(d=0.131)$. We know those three boundary equilibria $E_{0}(0,0), E_{1}(1,0)$, and $E_{2}(2 / 3,0)$ always exist no matter what the value of $d$ is. Thus, in the following cases we do not introduce the existence of boundary equilibria separately. However, if the value of $d$ is gradually increasing across the transcritical bifurcation TC1 and finally enters into region II, model (4a) and (4b) has an interior equilibrium point $E_{3}$. It is worth emphasizing that the critical value of transcritical bifurcation is $d_{\mathrm{TC} 1}=$ $e /(b+1)=0.13167$ and the interior equilibrium point $E_{3}(0.97064,0.039763)$ is a saddle point, boundary equilibria $E_{0}$ and $E_{1}$ are stable, and $E_{2}$ is an unstable node point, which has been shown in Figure 2(b) $(d=0.132)$. As the value of $d$ gradually increases to $d_{\mathrm{TC} 2}=e /(b m+1)+s(1-1 / m)=$ 0.132083 , which is a critical value of another transcritical bifurcation and finally enters into region III, model (4a) and (4b) has two interior equilibria $E_{3}(0.91328,0.093458)$ and $E_{4}(0.71172,0.052831)$, which are unstable; $E_{0}$ and $E_{1}$ are stable, and $E_{2}$ is a saddle point (see Figure 2(c), $d=0.1325$ ).

To further fully explore the existence of the equilibria and the occurrence of the bifurcation behavior in model (4a) and (4b), we take another set of parameter values in Figure 1(b) and repeat the steps in Figure 1(a). The bifurcation diagram in Figure 1(b) has been depicted in detail as follows. It is obvious to find that model (4a) and (4b) has an interior equilibrium point $E_{4}$ if the value of $d$ exceeds the threshold value $d_{\mathrm{TC} 3}=$ $e /(b m+1)+s(1-1 / m)=0.134583$, which is transcritical bifurcation threshold TC3. It is worthwhile to point out that the interior equilibrium point $E_{4}(0.69128,0.030678)$ is unstable on the account of $n_{4}=0.69128 \notin L(0.83822,1)$. The boundary equilibrium $E_{0}(0,0)$ is stable, and $E_{1}(1,0)$ and $E_{2}(2 / 3,0)$ are saddle points (see Figure $2(\mathrm{~d}), d=0.1349$ ). We take another set of parameters values as the supplement to display the case that there exists only one interior equilibrium point and it is locally asymptotically stable (see Figure 3(a)), where $E_{1}(1,0)$ and $E_{2}(2 / 3,0)$ are saddle points, $E_{0}(0,0)$ and $E_{4}(0.886762,0.040628)$ are stable, and $L=(0.84652,1)$. Furthermore, if the value of $d$ increases beyond the threshold value of transcritical bifurcation threshold $d_{\mathrm{TC} 4}=e /(b+$ $1)=0.135$ and finally enters into region $\mathrm{V}$, te model $(4 \mathrm{a})$ and $(4 \mathrm{~b})$ has three boundary equilibria $E_{0}(0,0), E_{1}(1,0)$, and $E_{2}(2 / 3,0)$ and two interior equilibria $E_{3}(0.97862,0.029801)$ and $E_{4}(0.71938,0.060341)$ (see Figure $\left.2(\mathrm{e}), d=0.1352\right)$. It is necessary to underline that $E_{0}(0,0)$ and $E_{1}(1,0)$ are stable and $n_{4}=0.71938 \notin L(0.83822,1)$, so the interior equilibria $E_{4}$ and $E_{3}$ are all unstable.

In order to clearly explore the steady characteristic of the interior equilibrium point $E_{4}$, Figure $1(\mathrm{c})$ will be given, which is the partially enlarged view of Figure 1(b). It should be stressed that the interior equilibrium $E_{4}$ will change the stable state if the value of $d$ increased beyond the line L1 and finally enters into region VI, which suggests that a Hopf bifurcation can lead to the appearance of a limit cycle in the vicinity of the interior equilibrium $E_{4}(0.83816,0.11816)$ if $d=0.135787825659$ (see Figure 2(f)). From Figure 2(f), we can observe that this limit cycle around $E_{4}$ is stable as it attracts two neighboring trajectories: the trajectory (bottle green curve) lying inside the limit cycle and the trajectory (blue curve) lying outside; these two trajectories move ectad and entad, respectively, and converge on the limit cycle. But at this time the interior equilibrium point $E_{3}(0.85397,0.11709)$ and boundary equilibrium point $E_{2}(2 / 3,0)$ are unstable, and $E_{0}(0,0)$ and $E_{1}(1,0)$ are stable. When the value of $d$ enters into domain VI, $E_{4}(0.86298,0.042889)$ becomes stable, $E_{3}(0.92702,0.032122)$ remains unstable, and $E_{0}$ and $E_{1}$ are all stable in this domain. Thus, it is interesting to know that model (4a) and (4b) will show three stationary phenomenon (see Figure $2(\mathrm{~g}), d=0.13579)$. Due to the parameter values, the nature of $E_{3}$ and $E_{4}$ only can be seen clearly on the enlarged view (see Figure 2(g)). Therefore, we take another group of values to exhibit the three stable states in overall view in Figure 3(b). If the value of $d$ gradually increases and reaches $d_{\mathrm{SN}}=b s+e+2 \sqrt{b s e}+s=0.1357900212$, it is easy to find that $E_{3}(0.84605,0.11789)$ and $E_{4}(0.84605,0.11789)$ coincide at line L2 and the coincident point is called saddlenode point, which has features of both saddle and node points (see Figure 2(h)). However, it will disappear if the value of $d$ is increased higher than $d_{\mathrm{SN}}$, which signifies that model (4a) and (4b) will undergo a saddle-node bifurcation if the value of $d$ increases across the threshold value $d_{\mathrm{SN}}=b s+e+2 \sqrt{b s e}+s$. In a word, it is worthy of our summary that model (4a) and (4b) can show a complex dynamic evolutionary process of steady conversion and bifurcation behavior with increase of key parameter $d$.

In addition, model (4a) and (4b) has three boundary equilibria $E_{0}(0,0), E_{1}(1,0)$, and $E_{2}(2 / 3,0)$ and an interior equilibrium point $E_{3}$, which is unstable if $s>d$ or $s=d$. However, it is worth stressing that the boundary equilibrium point $E_{0}(0,0)$ is a saddle point if $s>d$ and is an unstable high-order singularity if $s=d$ (see Figures 4(a) and 4(b)). At the same time, it can be known from Theorem 7 that model (4a) and (4b) can undergo a Bogdanov-Takens bifurcation if $\left.\operatorname{Tr}\left(J_{E^{*}}\right)\right|_{\left(e_{\mathrm{BT}}, b_{\mathrm{BT}}\right)}=0$ and $\left.\operatorname{Det}\left(J_{E^{*}}\right)\right|_{\left(e_{\mathrm{BT}}, b_{\mathrm{BT}}\right)}=0$, which is shown in Figure 4(c).

Based on the above analysis, the key parameter $d$ can impose influence on dynamic evolutionary mechanism of steady conversion and bifurcation behavior and lead to model (4a) and (4b) having three stationary phenomena and multiple bifurcation behaviors, which can in turn prove that the theoretical results are correct and the complex dynamics of model (4a) and (4b) mainly depend on some key parameters. Moreover, these results show that the method of using mathematical model to study the ecological problems is feasible.

\section{Conclusions}

On the basis of the theories and methods of ecology, a predator-prey model is studied numerically and analytically in this paper. The aim is to probe how some key factors influence dynamic evolutionary mechanism of steady conversion and bifurcation behavior in predator-prey model. The theoretical works have been promoting the investigation of the existence and stability of the equilibria, as well as some conditions of some bifurcations behaviors, such as transcritical bifurcation, saddle-node bifurcation, and Hopf 


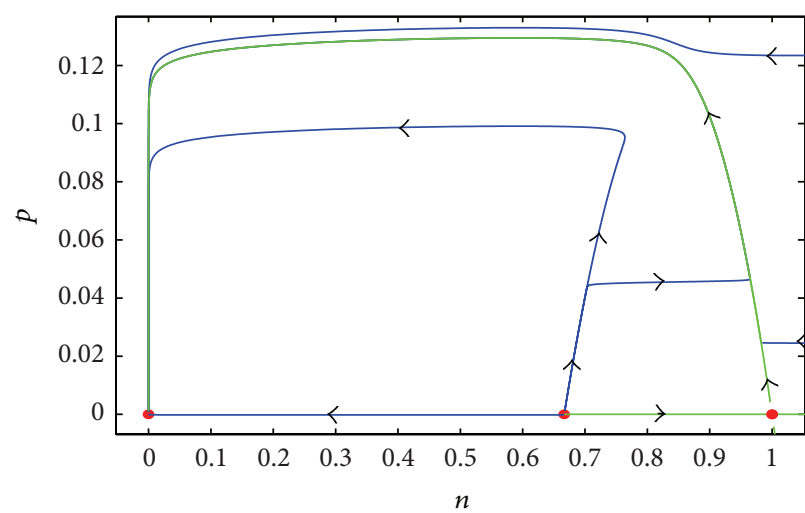

(a)

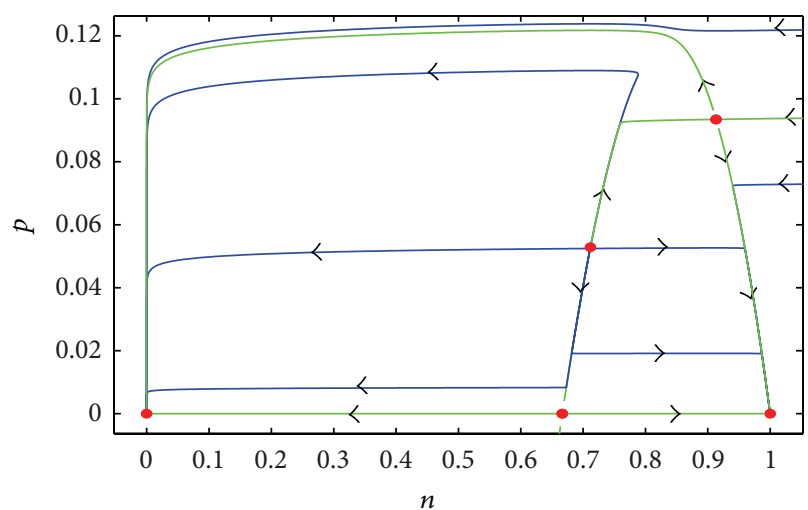

(c)

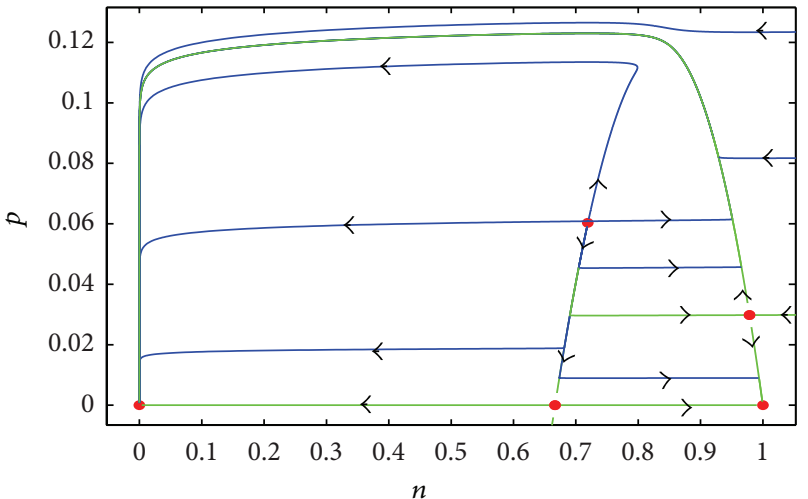

(e)

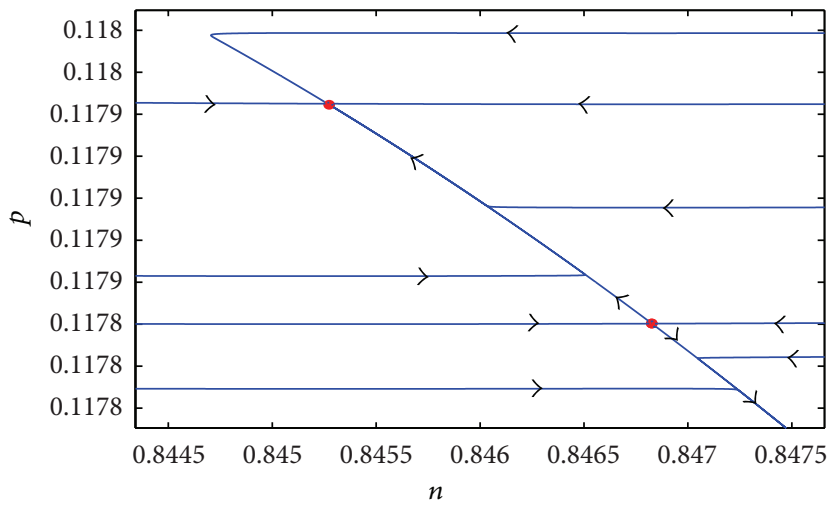

(g)

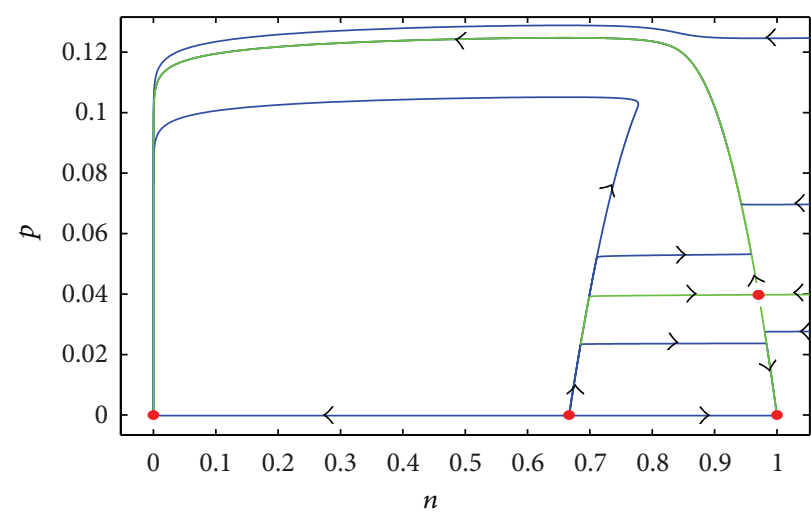

(b)

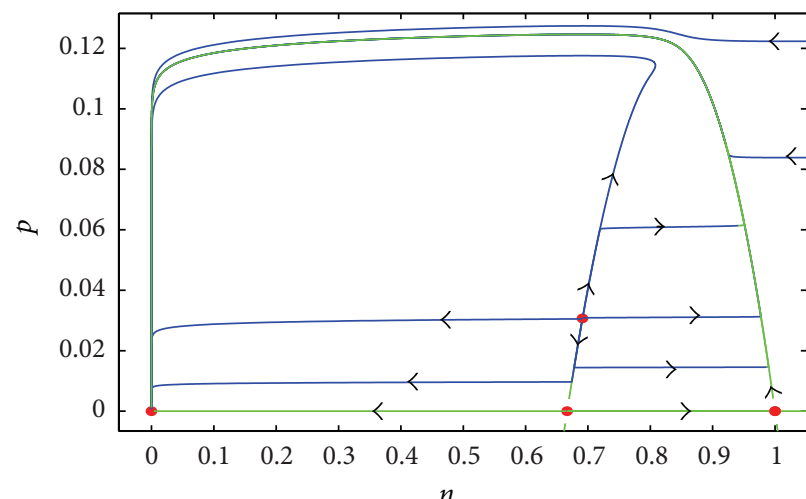

(d)

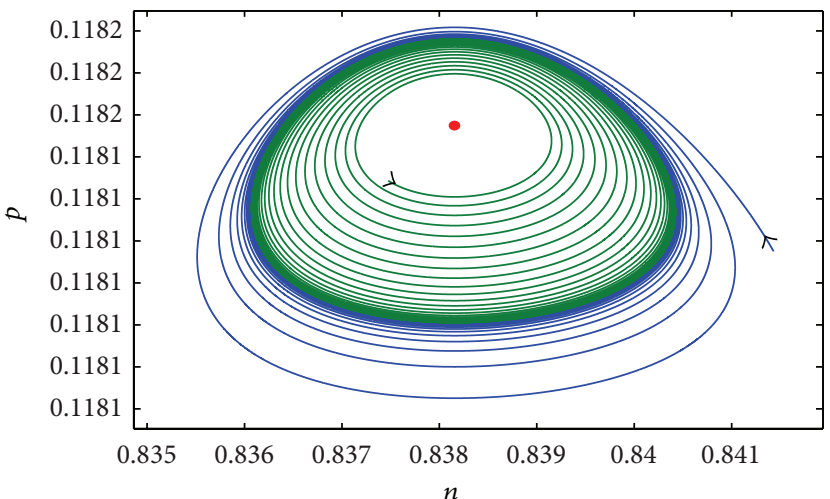

(f)

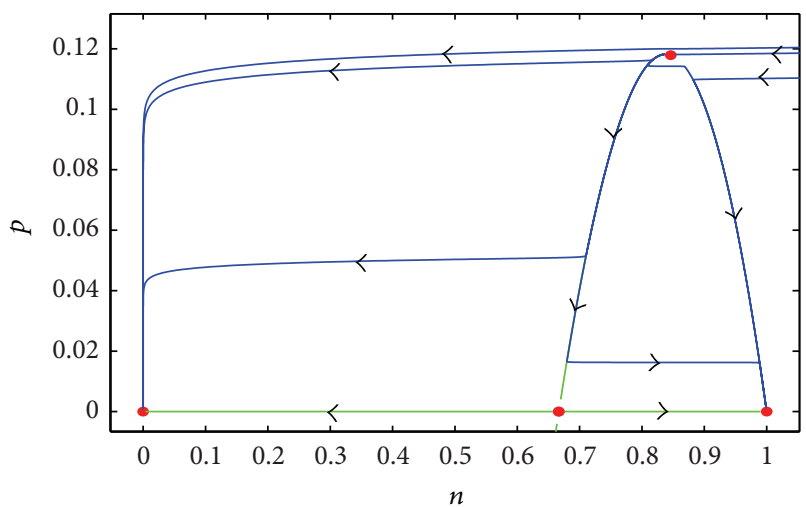

(h)

FIGURE 2: Phase portraits for different concomitant case of the model's equilibria. The horizontal axis is prey population $n$ and the vertical axis is predator population $p$. The green curves are stable or unstable orbits; the red point is the equilibrium point. We take $m=1.5, b=2.0$, $s=0.1, e=0.395$ in $(\mathrm{a}-\mathrm{c})$, and $e=0.405$ in $(\mathrm{d}-\mathrm{h})$. 


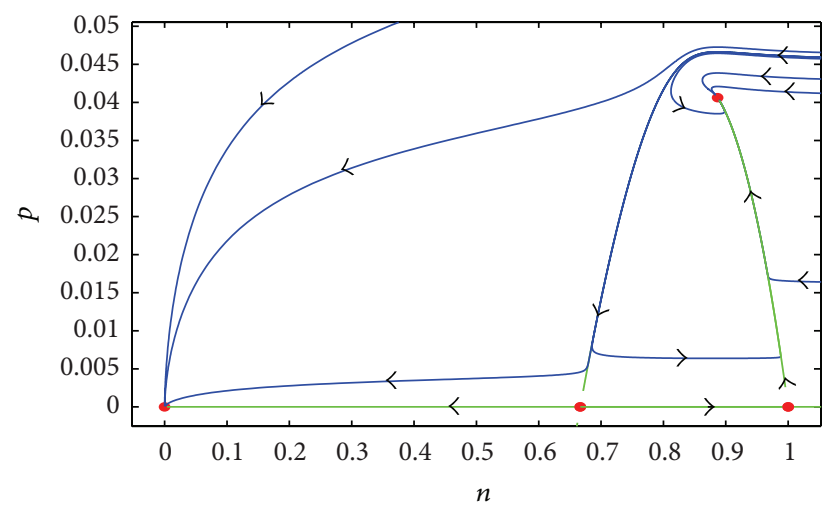

(a)

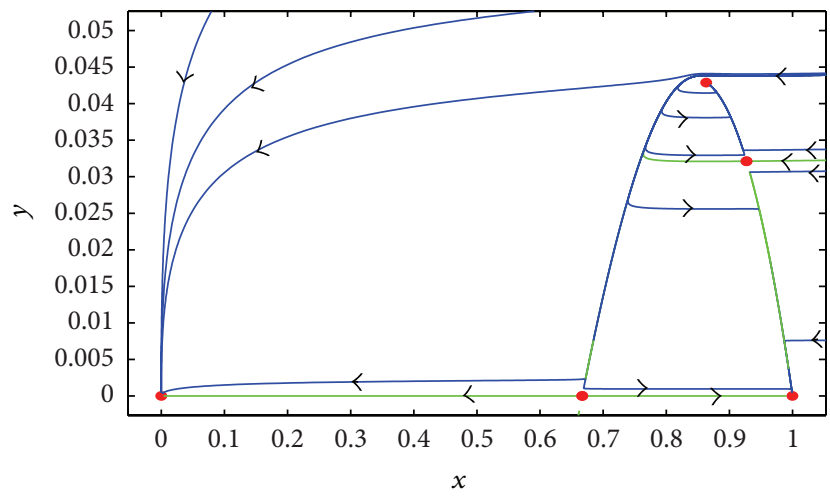

(b)

Figure 3: Phase portraits for $m=1.5, s=0.1$, and $b=0.2$; (a) $e=0.844, d=0.7$; (b) $e=0.599, d=0.5$.

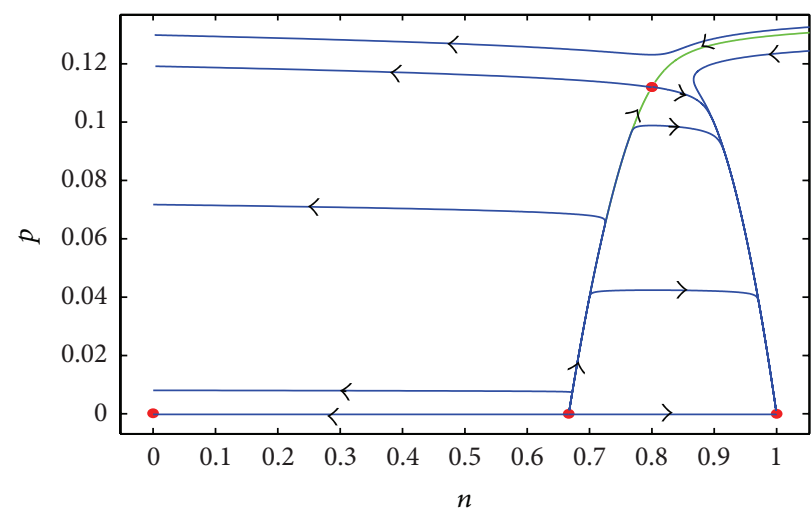

(a)

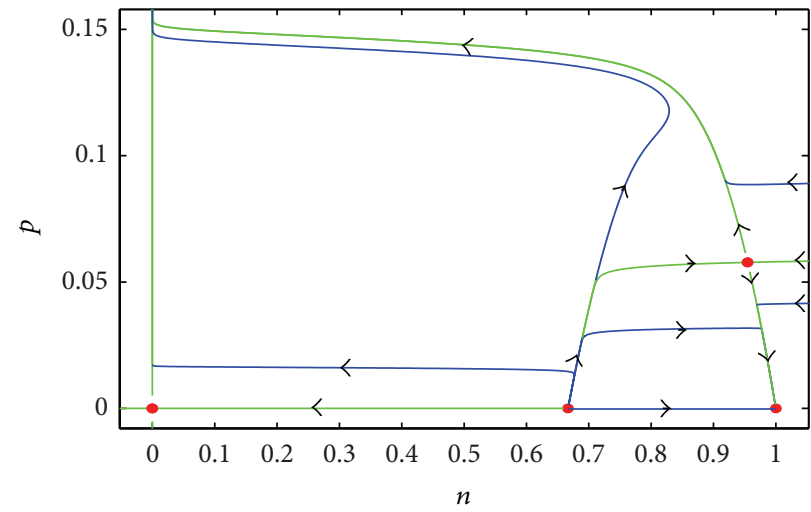

(b)

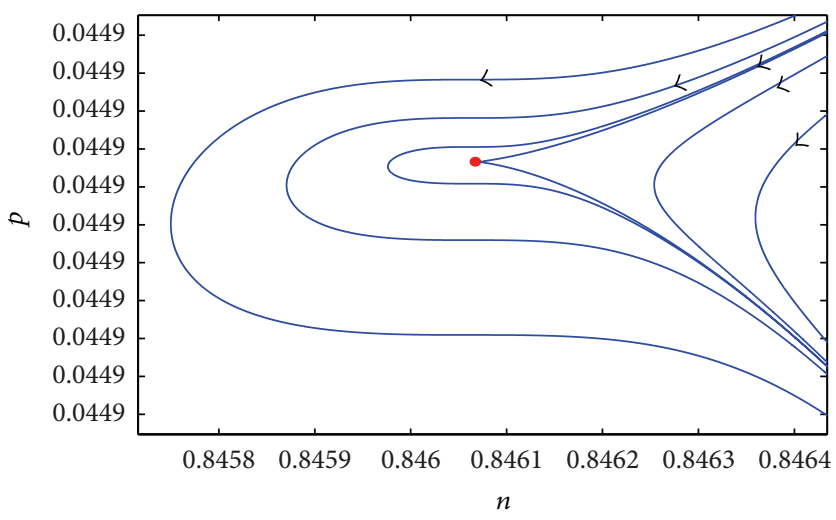

(c)

FIGURE 4: Phase portraits for $m=1.5, s=0.1, e=0.28, b=2.0$, (a) $d=0.1$, and (b) $d=0.095$. The parameter values in image (c) are introduced in Theorem 7.

bifurcation, which can deduce a standard parameter controlled relationship and in turn provide a theoretical basis for the numerical simulation.

Numerical analysis indicates that the dynamic evolutionary mechanism of steady conversion and bifurcation behavior mainly depend on a specific key parameter $d$. Within this framework, the direct and indirect effects caused by the specific key parameter $d$ are investigated by means of bifurcation analysis and phase diagram. It is obvious to find that the existence and stability of interior equilibria $E_{3}$ and $E_{4}$ mainly depend on a key parameter $d$. These results suggest that the key parameter $d$ plays an important role in the prey-predator model. In addition, when the value of the key parameter $d$ is larger than some critical value, the model can possess multiple bifurcation behaviors, such as transcritical bifurcation, saddle-node bifurcation, and Hopf 
bifurcation. Thus, it is worthwhile to remark that the key parameter $d$ has a profound effect on the population bifurcation dynamical behaviors. In a word, some key parameters can alter population dynamics and features in prey-predator model. In addition, it is our hope that all these results can be applied in the study of the dynamic complexity of ecosystems.

\section{Conflict of Interests}

The authors declare that there is no conflict of interests regarding the publication of this paper.

\section{Acknowledgments}

This work was supported by the National Key Basic Research Program of China (973 Program, Grant no. 2012CB426510), by the National Natural Science Foundation of China (Grant no. 31370381), and by the Key Program of Zhejiang Provincial Natural Science Foundation of China (Grant no. LZ12C03001).

\section{References}

[1] H. N. Comins and D. W. E. Blatt, "Prey-predator models in spatially heterogeneous environments," Journal of Theoretical Biology, vol. 48, no. 1, pp. 75-83, 1974.

[2] H. N. Agiza, E. M. ELabbasy, H. E. Metwally, and A. A. Elsadany, "Chaotic dynamics of a discrete prey-predator model with Holling type II," Nonlinear Analysis. Real World Applications, vol. 10, no. 1, pp. 116-129, 2009.

[3] K. Chakraborty, S. Jana, and T. K. Kar, "Global dynamics and bifurcation in a stage structured prey-predator fishery model with harvesting," Applied Mathematics and Computation, vol. 218, no. 18, pp. 9271-9290, 2012.

[4] Z. H. Lu, X. B. Chi, and L. S. Chen, "Impulsive control strategies in biological control of pesticide," Theoretical Population Biology, vol. 64, no. 1, pp. 39-47, 2003.

[5] Y. N. Xiao and L. S. Chen, "Modeling and analysis of a predatorprey model with disease in the prey," Mathematical Biosciences, vol. 171, no. 1, pp. 59-82, 2001.

[6] T. K. Kar, "Stability analysis of a prey-predator model incorporating a prey refuge," Communications in Nonlinear Science and Numerical Simulation, vol. 10, no. 6, pp. 681-691, 2005.

[7] Y. J. Huang, F. D. Chen, and L. Zhong, "Stability analysis of a prey-predator model with Holling type III response function incorporating a prey refuge," Applied Mathematics and Computation, vol. 182, no. 1, pp. 672-683, 2006.

[8] T. Legović and A. Cruzado, "A model of phytoplankton growth on multiple nutrients based on the Michaelis-Menten-Monod uptake, Droop's growth and Liebig's law," Ecological Modelling, vol. 99, no. 1, pp. 19-31, 1997.

[9] X. H. Wang and J. W. Jia, "Dynamic of a delayed predatorprey model with birth pulse and impulsive harvesting in a polluted environment," Physica A: Statistical Mechanics and its Applications, vol. 422, pp. 1-15, 2015.

[10] K. Das and N. H. Gazi, "Random excitations in modelling of algal blooms in estuarine systems," Ecological Modelling, vol. 222, no. 14, pp. 2495-2501, 2011.

[11] J. Yang and M. Zhao, "Complex behavior in a fish algae consumption model with impulsive control strategy," Discrete
Dynamics in Nature and Society, vol. 2011, Article ID 163541, 17 pages, 2011.

[12] E. González-Olivares and A. Rojas-Palma, "Allee effect in Gause type predator-prey models: existence of multiple attractors, limit cycles and separatrix curves. A brief review," Mathematical Modelling of Natural Phenomena, vol. 8, no. 6, pp. 143-164, 2013.

[13] J. Dhar, R. S. Baghel, and A. K. Sharma, "Role of instant nutrient replenishment on plankton dynamics with diffusion in a closed system: a pattern formation," Applied Mathematics and Computation, vol. 218, no. 17, pp. 8925-8936, 2012.

[14] J. H. Luo, "Phytoplankton-zooplankton dynamics in periodic environments taking into account eutrophication," Mathematical Biosciences, vol. 245, no. 2, pp. 126-136, 2013.

[15] R. R. Vance, "Predation and resource partitioning in one predator-two prey model communications," The American Naturalist, vol. 112, no. 987, pp. 797-813, 1978.

[16] H. Cornell, "Search strategies and the adaptive significance of switching in some general predators," The American Naturalist, vol. 110, no. 972, pp. 317-320, 1976.

[17] O. P. Misra, P. Sinha, and C. S. Sikarwar, "Dynamical study of a prey-predator system with a commensal species competing with prey species: effect of time lag and alternative food source," Computational and Applied Mathematics, vol. 34, no. 1, pp. 343361, 2015.

[18] X. H. Zhang, W. X. Li, M. Liu, and K. Wang, "Dynamics of a stochastic Holling II one-predator two-prey system with jumps," Physical A: Statistical Mechanics and Its Applications, vol. 421, pp. 571-582, 2015.

[19] M. van Baalen, V. Křivan, P. C. J. van Rijn, and M. W. Sabelis, "Alternative food, switching predators, and the persistence of predator-prey systems," The American Naturalist, vol. 157, no. 5, pp. 512-524, 2001.

[20] P. D. Spencer and J. S. Collie, "A simple predator-prey model of exploited marine fish populations incorporating alternative prey," ICES Journal of Marine Science, vol. 53, no. 3, pp. 615-628, 1996.

[21] W. W. Zhang and M. Zhao, "Dynamical complexity of a spatial phytoplankton-zooplankton model with an alternative prey and refuge effect," Journal of Applied Mathematics, vol. 2013, Article ID 608073, 10 pages, 2013.

[22] T. K. Kar and S. K. Chattopadhyay, "A focus on long-run sustainability of a harvested prey predator system in the presence of alternative prey," Comptes Rendus-Biologies, vol. 333, no. 1112, pp. 841-849, 2010.

[23] C. S. Holling, "The functional response of predators to prey density and its role in mimicry and population regulation," Memoirs of the Entomological Society of Canada, vol. 97, supplement 45, pp. 5-60, 1965.

[24] C. J. Dai and M. Zhao, "Mathematical and dynamic analysis of a prey-predator model in the presence of alternative prey with impulsive state feedback control," Discrete Dynamics in Nature and Society, vol. 2012, Article ID 724014, 19 pages, 2012.

[25] W. C. Allee and E. S. Bowen, "Studies in animal aggregations: mass protection against colloidal silver among goldfishes," Journal of Experimental Zoology, vol. 61, no. 2, pp. 185-207, 1932.

[26] S.-R. Zhou, Y.-F. Liu, and G. Wang, "The stability of predatorprey systems subject to the Allee effects," Theoretical Population Biology, vol. 67, no. 1, pp. 23-31, 2005.

[27] J. F. Wang, J. P. Shi, and J. P. Wei, "Predator-prey system with strong Allee effect in prey," Journal of Mathematical Biology, vol. 62, no. 3, pp. 291-331, 2011. 
[28] W.-M. Wang, Y.-N. Zhu, Y.-L. Cai, and W.-J. Wang, "Dynamical complexity induced by Allee effect in a predator-prey model," Nonlinear Analysis: Real World Applications, vol. 16, pp. 103-119, 2014.

[29] A. M. Kramer, B. Dennis, A. M. Liebhold, and J. M. Drake, “The evidence for Allee effects," Population Ecology, vol. 51, no. 3, pp. 341-354, 2009.

[30] M.-H. Wang and M. Kot, "Speeds of invasion in a model with strong or weak Allee effects," Mathematical Biosciences, vol. 171, no. 1, pp. 83-97, 2001.

[31] E. Aulisa and S. R. J. Jang, "Continuous-time predator-prey systems with Allee effects in the prey," Mathematics and Computers in Simulation, vol. 105, pp. 1-16, 2014.

[32] X. H. Pan, M. Zhao, Y. P. Wang, H. Yu, Z. Ma, and Q. Wang, "Stability and dynamical analysis of a biological system," Abstract and Applied Analysis, vol. 2014, Article ID 952840, 11 pages, 2014.

[33] J. Sotomayor, "Generic bifurcations of dynamical systems," Dynamical Systems, pp. 549-560, 1973.

[34] L. Perko, Differential Equations and Dynamical Systems, Springer, Berlin, Germany, 2000.

[35] B. D. Hassard, N. D. Kazarinoff, and Y. H. Wan, Theory and Applications of Hopf Bifurcation, Cambridge University Press, London, UK, 1981.

[36] D. M. Xiao and S. G. Ruan, "Bogdanov-Takens bifurcations in predator-prey systems with constant rate harvesting," Fields Institute Communications, vol. 21, pp. 493-506, 1999.

[37] R. I. Bogdanov, "Bifurcations of a limit cycle for a family of vector fields on the plane," Selecta Mathematica, vol. 1, pp. 373388, 1981.

[38] J. C. Polking, Phase Plane pplane8.m (MATLAB ${ }^{\circledR}$ Toolbox), Department of Mathematics, Rice University, 2009, http://math.rice.edu/ dfield/\#8.0.

[39] A. Regalado-Méndez and J. G. Madrid-González, "Dynamic system analysis with pplane8.m (MATLAB ${ }^{\circledR}$ toolbox)," Avances en Ciencias e Ingeniería, pp. 117-132, 2013. 


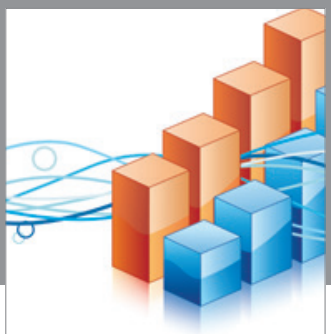

Advances in

Operations Research

vatem alat4

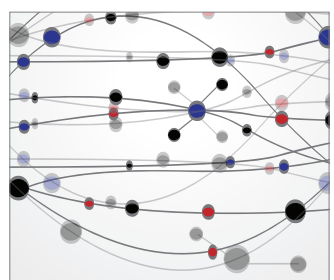

\section{The Scientific} World Journal
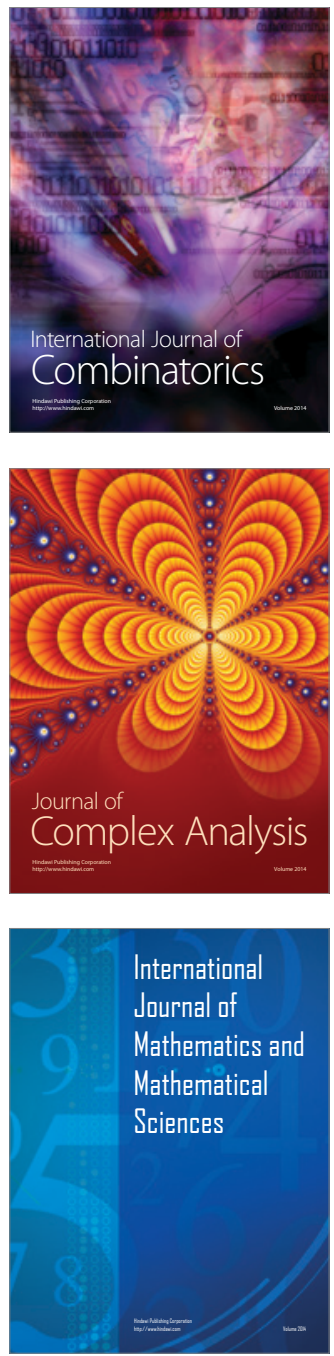
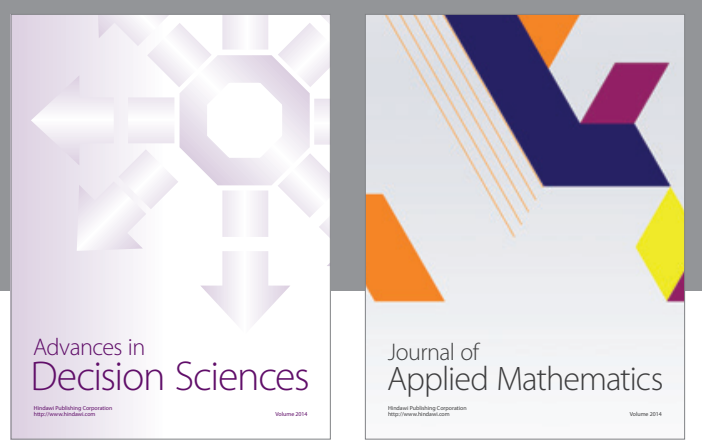

Algebra

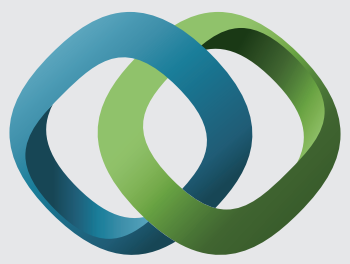

\section{Hindawi}

Submit your manuscripts at

http://www.hindawi.com
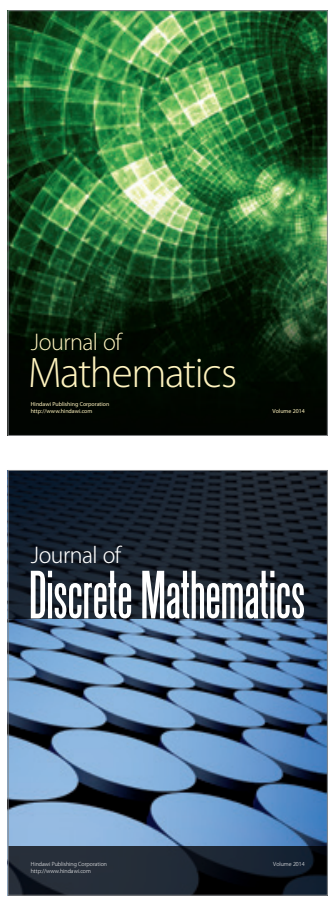

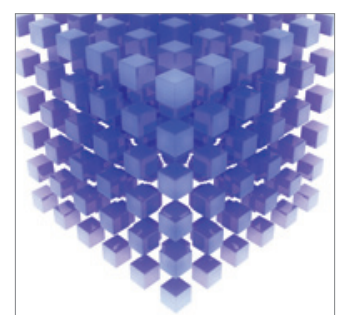

Mathematical Problems in Engineering
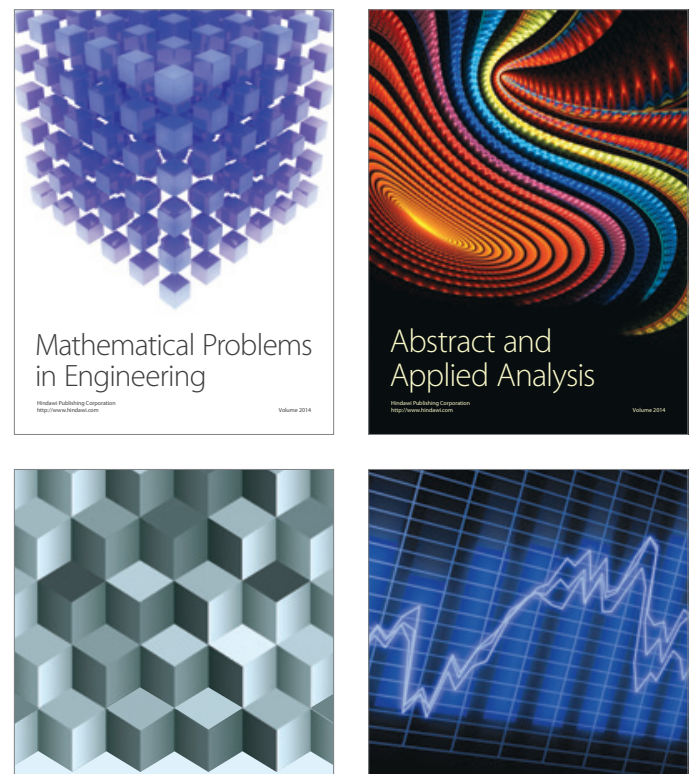

Journal of

Function Spaces

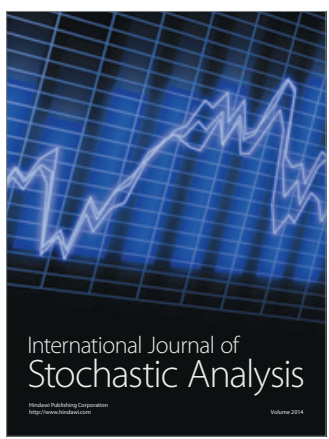

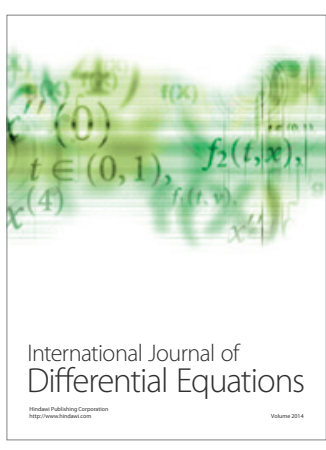
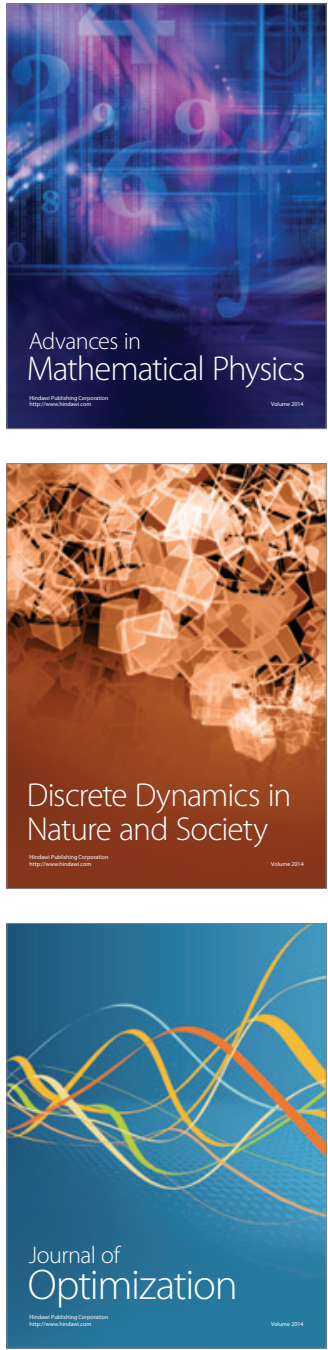